\title{
ESTUDO DEMOGRÁFICO DE PACIENTES PORTADORES DE DEFORMIDADES DE COLUNA VERTEBRAL QUE AGUARDAM CIRURGIA EM HOSPITAL TERCIÁRIO DE ALTA COMPLEXIDADE
}

\author{
DEMOGRAPHIC STUDY OF PATIENTS WITH SPINAL DEFORMITIES WHO ARE AWAITING \\ SURGERY IN A TERTIARY HOSPITAL OF HIGH COMPLEXITY
}

\author{
ESTUDIO DEMOGRÁFICO DE PACIENTES CON DEFORMIDADES DE LA COLUMNA \\ EN ESPERA DE CIRUGÍA EN HOSPITAL DE TERCER NIVEL DE ALTA COMPLEJIDAD
}

Nicola Jorge Carneiro Neto ${ }^{1}$, Ricardo Umeta ${ }^{2}$, Robert Meves ${ }^{3}$, Maria Fernanda Silber Caffaro ${ }^{4}$, Élcio Landim ${ }^{5}$, Osmar Avanzi ${ }^{6}$

\begin{abstract}
RESUMO
Objetivo: Definir as características demográficas dos pacientes portadores de deformidades secundárias da coluna vertebral que aguardam em fila de espera para tratamento cirúrgico em hospital terciário de alta complexidade. Métodos: As informações foram obtidas através dos prontuários dos pacientes no período previamente definido. Os dados foram selecionados conforme os critérios já apresentados e manipulados estatisticamente. Resultados: O estudo incluiu um total de 170 pacientes, dos quais 94 eram do sexo feminino com idades entre 1 e 58 anos, e 76 do sexo masculino com idade entre 1 e 26 anos. Entre as deformidades encontradas foram identificadas: escolioses congênitas por segmentação (6 ou 3,5\%), formação (39 ou 22,9\%), mistas (34 ou 20,0\%) e complexas (14 ou 8,2\%), escolioses neuromusculares por paralisia cerebral (PC) (54 ou 31,8\%), miopatia (11 ou 6,5\%), artrogripose (2 ou 1,2\%) e escolioses por neurofibromatose (10 ou 5,9\%). Conclusão: Existe uma considerável lista de pacientes portadores de deformidades de coluna vertebral que aguardam em fila de espera para tratamento cirúrgico por um período muitas vezes bastante prolongado. Este fato acarreta um problema social de difícil manuseio e quando somadas, escoliose neuromuscular e escoliose congênita são responsáveis pela grande maioria dos casos que aguardam cirurgia.
\end{abstract}

Descritores: Coluna vertebral/anatomia \& histologia; Coluna vertebral/cirurgia; Anormalidades congênitas.

\begin{abstract}
Objective: Set the demographic characteristics of patients with secondary deformities of the spine waiting for surgical treatment in a tertiary hospital of high complexity. Methods: Information was obtained from patient charts in the predefined period. The data were selected according to the criteria already presented and manipulated statistically. Results The study included a total of 170 patients, of which 94 were female aged between 1 and 58 years, and 76 males aged between 1 and 26 years. Among the deformities found the following were identified: congenital scoliosis were identified by segmentation (6 or 3.5\%), formation (39 or 22.9\%), mixed (34 or $20.0 \%$ ) and complex (14 or $8.2 \%)$, neuromuscular scoliosis in cerebral palsy (PC) (54 or 31.8\%), myopathy (11 or 6.5\%), arthrogryposis (2 or 1.2\%) and scoliosis in neurofibromatosis (10 or 5.9\%). Conclusion There is a considerable list of patients with spinal deformities waiting for surgical treatment for a period of time often quite prolonged. This fact leads to a social problem difficult to handle and when added together, neuromuscular scoliosis and congenital scoliosis are responsible for the vast majority of cases awaiting surgery.
\end{abstract}

Keywords: Spine/anatomy \& histology; Spine/surgery; Congenital abnormalities.

RESUMEN

Objetivo: Objetivo: Definir las características demográficas de los pacientes con deformidades secundarias de la columna vertebral en la lista de espera para el tratamiento quirúrgico en un hospital de tercer nivel de complejidad alta. Métodos: Las informaciones fueron obtenidas de los archivos de pacientes en período predefinido. Los datos fueron seleccionados de acuerdo con los criterios ya presentados y manipulados estadísticamente. Resultados: En el estudio participaron un total de 170 pacientes, de los cuales 94 eran mujeres de edades comprendidas entre 1 y 58, y 76 varones de edades comprendidas entre 1 y 26 años. Entre las malformaciones encontradas fueron identificadas: escoliosis congénita por segmentación (6 o 3,5\%), formación (39 o 22,9\%), mixta (34 o 20,0\%) y compleja (14 o $8.2 \%)$, escoliosis neuromuscular en la parálisis cerebral (PC) (54 o 31,8\%), miopatía (11 o 6,5\%), artrogriposis (2 o 1,2\%) y escoliosis por neurofibromatosis (10 o 5,9\%). Conclusión Hay una lista considerable de pacientes con deformidades de la columna que aguardan en lista de espera por el tratamiento quirúrgico a menudo durante un período bastante prolongado. Este hecho produce un problema social difícil de manejar cuando se añaden escoliosis neuromuscular y escoliosis congénita, es responsable de la gran mayoría de los casos en la espera de la cirugía.

Descriptores: Spine/anatomía \& histologia; Spine/cirugía; Anomalías congénitas.

\footnotetext{
1. Médico Estagiário do Grupo de Cirurgia da Coluna do Departamento Ortopedia eTraumatologia da Faculdade de Ciências Médicas da Santa Casa de São Paulo - FCMSCSP - São Paulo (SP), Brasil. 2. Médico Pós-graduando do Departamento Ortopedia eTraumatologia da Faculdade de Ciências Médicas da Santa Casa de São Paulo - FCMSCSP - São Paulo (SP), Brasil.

3. Doutor, Professor e Chefe do Grupo de Coluna do Departamento Ortopedia e Traumatologia da Faculdade de Ciências Médicas da Santa Casa de São Paulo - FCMSCSP - São Paulo (SP), Brasil.

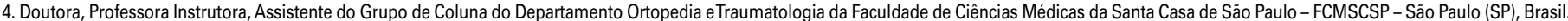

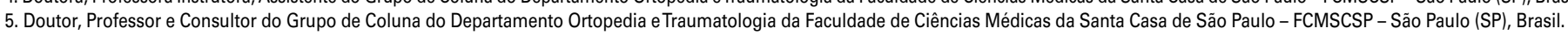

6. Doutor, Professor Adjunto e Diretor do Departamento Ortopedia eTraumatologia da Faculdade de Ciências Médicas da Santa Casa de São Paulo - FCMSCSP - São Paulo (SP), Brasil.
}

Trabalho realizado no Departamento de Ortopedia eTraumatologia da Faculdade de Ciências Médicas da Santa Casa de Misericórdia de São Paulo, São Paulo, SP. Correspondência: Rua Dr Cesário Motta Jr, 61 - Vila Buarque - 1221020. São Paulo, SP. Brasil. nelsonan@yahoo.com; robertmeves@ hotmail.com 


\section{INTRODUÇÃO}

A coluna vertebral constitui a principal parte subcranial do esqueleto axial. Os seus elementos são unidos por uma série de articulações intervertebrais que formam um suporte flexível e capaz de proteger estruturas neurológicas. Geralmente é formada por 33 vértebras sendo dividida em segmento móvel (7 cervicais de C1 a C7, 12 torácicas de T1 a T12 e 5 lombares de L1 a L5) e segmento rígido (5 sacrais de $\mathrm{S} 1$ a S5 e 4 coccígeas) ${ }^{1}$. A coluna vertebral isenta de malformações é retilínea no plano anteroposterior, mas apresenta curvas fisiológicas quando vista no plano sagital. A cifose torácica média é de 30 a $35^{\circ}$ (variando entre 10 e $50^{\circ}$ ) e a lordose lombar apresenta curvatura média de 50 a $60^{\circ}$ (variando entre 35 e $\left.80^{\circ}\right)^{2,3}$.

Na maioria dos estudos publicados, as deformidades da coluna vertebral são caracterizadas pela ocorrência de desvio no plano coronal maior do que $10^{\circ 4-6}$. Elas podem ser divididas de acordo com a localização do ápice da curva, sendo classificadas em cervical (entre C2 e C6), cervicotorácica (entre C7 e T1), torácica (entre T2 e T11), toracolombar (entre T12 e L1), lombar (entre L2 e L4) e lombossacral (L5 ou abaixo) ${ }^{3}$. Também podem ser divididas de acordo com a idade de surgimento, sendo classificadas em infantil (de 0 a 3 anos), juvenil (de 4 a 10 anos), do adolescente (de 11 a 17 anos) e do adulto (igual ou maior a 18 anos) ${ }^{3}$.

Estas deformidades passam a apresentar indicação cirúrgica nos casos em que a manipulação da curva se torna de difícil controle com o uso de coletes ou quando o ângulo de Cobb supera $50^{\circ 7}$. Conforme Edgar e Weinstein, curvas deste porte tendem a progredir na vida adulta e apresentam como principais complicações falência cardiorespiratória que é diretamente proporcional a sua progressão e deformidade estética ${ }^{8}$.

No Brasil pacientes portadores de deformidades na coluna muitas vezes aguardam anos em filas de espera cirúrgica nos hospitais terciários de alta complexidade do SUS o que acarreta em um importante problema social.

Considerando a classificação etiológica proposta pela Scoliosis Research Society (SRS) ${ }^{9}$, também podem ser utilizadas as definições vistas no Quadro 1

Quadro 1. Classificação etiológica das deformidades da coluna vertebral conforme a Scoliosis Research Society ${ }^{9}$.

\begin{tabular}{|c|c|}
\hline Etiologia & Tipos \\
\hline Idiopática & Infantil, juvenil, do adolescente e do adulto. \\
\hline Neuropática & $\begin{array}{l}\text { Paralisia cerebral, siringomielia, tumor da medula, } \\
\text { poliomielite, mielomeningocele e outros. }\end{array}$ \\
\hline Miopática & Artrogripose, distrofia muscular de Duchenne e outros. \\
\hline Congênita & Defeito de formação, segmentação e misto. \\
\hline Tecido nervoso & Meningocele, disrafismo espinal e outros. \\
\hline \multicolumn{2}{|l|}{ Neurofibromatose } \\
\hline Mesenquimatosa & Sindrome de Marfan, síndrome de Ehlers-Danlos e outros. \\
\hline Traumática & Fratura ou luxação, pós-irradiação e outros. \\
\hline Contraturas de tecidos moles & Pós-empiema, queimaduras e outros. \\
\hline Osteocondrodistrofias & Acondroplasia, mucopolissacaridoses e outros. \\
\hline Tumoral & Benigna ou maligna. \\
\hline Doença reumatóide & Artrite reumatóide e outros. \\
\hline Metabólica & Raquitismo, osteogênese imperfeita e osteoporose juvenil. \\
\hline Relacionada à área lombo-sacral & Espondilólise, espondilolistese e outros. \\
\hline Toracogênica & Pós-toracoplastia ou toracotomia. \\
\hline \multicolumn{2}{|l|}{ Histérica } \\
\hline Funcional & Postural, por anisomelia e outros. \\
\hline
\end{tabular}

Neste trabalho foram selecionados pacientes portadores de deformidades secundárias na coluna vertebral que aguardam em fila de espera para tratamento cirúrgico no serviço de Ortopedia e Traumatologia da Santa Casa de Misericórdia de São Paulo.

\section{MATERIAIS E MÉTODOS}

\section{Materiais}

Foram selecionados 170 pacientes (94 do sexo feminino e 76 do sexo masculino), portadores de deformidades na coluna vertebral e incluídos na lista de espera do ambulatório de cirurgia da coluna do Departamento de Ortopedia e Traumatologia da Irmandade da Santa Casa de Misericórdia de São Paulo (DOT-ISCMSP), no período compreendido entre Janeiro 2002 e Dezembro de 2009. Os pacientes apresentaram faixa etária entre 1 e 58 anos, com média de 15,3 anos.

Foram considerados critérios de inclusão, pacientes com deformidades secundárias na coluna vertebral e indicação de tratamento cirúrgico. Como critérios de exclusão, foram descartados os pacientes portadores de escolioses idiopáticas, sem indicação de tratamento cirúrgico, submetidos a tratamento cirúrgico prévio ou aqueles que não concordaram em participar do estudo.

Esta pesquisa foi aprovada pelo Comitê de Ética em Pesquisa em Seres Humanos do DOT-ISCMSP sob o número 120/10.

\section{MÉTODOS}

As informações constantes neste estudo foram obtidas através dos prontuários dos pacientes do Serviço de Arquivos Médicos e Estatístico (SAME-ISCMSP) no período previamente definido. Os dados foram selecionados conforme os critérios já apresentados e manipulados estatisticamente utilizando-se os programas Excel for Windows ${ }^{\circledR}$ versão 2003 e SPSS ${ }^{\circledR}$ versão 10.0.

\section{OBJETIVOS}

Definir as características demográficas dos pacientes portadores de deformidades secundárias da coluna vertebral que aguardam em fila de espera para tratamento cirúrgico em hospital terciário de alta complexidade

\section{RESULTADOS}

O estudo incluiu um total de 170 pacientes, dos quais 94 eram do sexo feminino com idades entre 1 e 58 anos, e 76 do sexo masculino com idade entre 1 e 26 anos. Esta distribuição por gênero está representada na Figura 1.

Considerando a amostragem utilizada no presente estudo, foi efetuada a divisão dos pacientes considerando-se o gênero

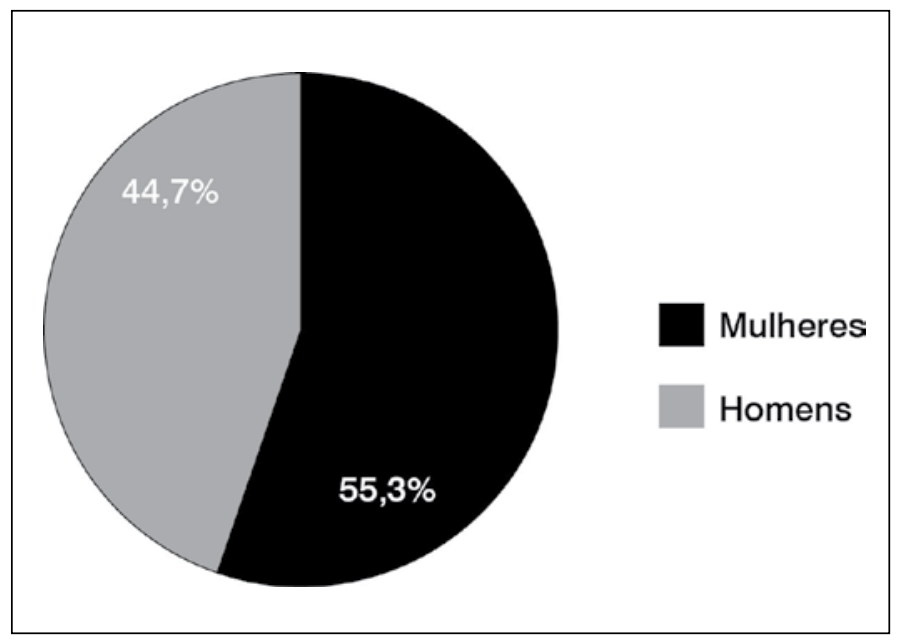

Figura 1. Distribuição dos pacientes por gênero. 
e a faixa etária. Para ambos os gêneros, a moda situou-se na faixa entre 10 (inclusive) e 15 (exclusive) anos, conforme visto na Figura 2. Para melhor visualização do gráfico de distribuições, foram amputadas as faixas acima de 35 anos, pois as mesmas incluiriam apenas 2 casos do sexo feminino, sendo 1 na faixa 4045 e outro na faixa 55-60 anos. A moda obtida foi bastante baixa, fato que pode ser associado à desistência do acompanhamento ambulatorial pelos pacientes mais idosos ou familiares ou pelo óbito devido às complicações relacionadas à deformidade.

Dentre as deformidades encontradas neste estudo, foram identificadas escolioses congênitas por segmentação (6 ou 3,5\%), formação (39 ou 22,9\%), mistas (34 ou 20,0\%) e complexas (14 ou 8,2\%), escolioses neuromusculares por paralisia cerebral ou PC (54 ou $31,8 \%$ ), miopatia (11 ou 6,5\%), artrogripose (2 ou 1,2\%) e escolioses por neurofibromatose (10 ou 5,9\%), conforme demonstrado na Figura 3. A divisão das escolioses congênitas em 4 sub-grupos adotada no presente estudo seguiu o padrão indicado por Winter et al. ${ }^{10}$.

Pode ser observado que, na amostragem utilizada, a causa mais frequente de deformidade secundária foi escoliose neuromuscular por PC.

Quando somadas, as escolioses congênitas foram responsáveis por $54,6 \%$ dos casos analisados no presente trabalho. A Figura 4 demonstra a divisão das escolioses congênitas em pacientes com até 10 anos de idade e pacientes com idade superior a esse valor.

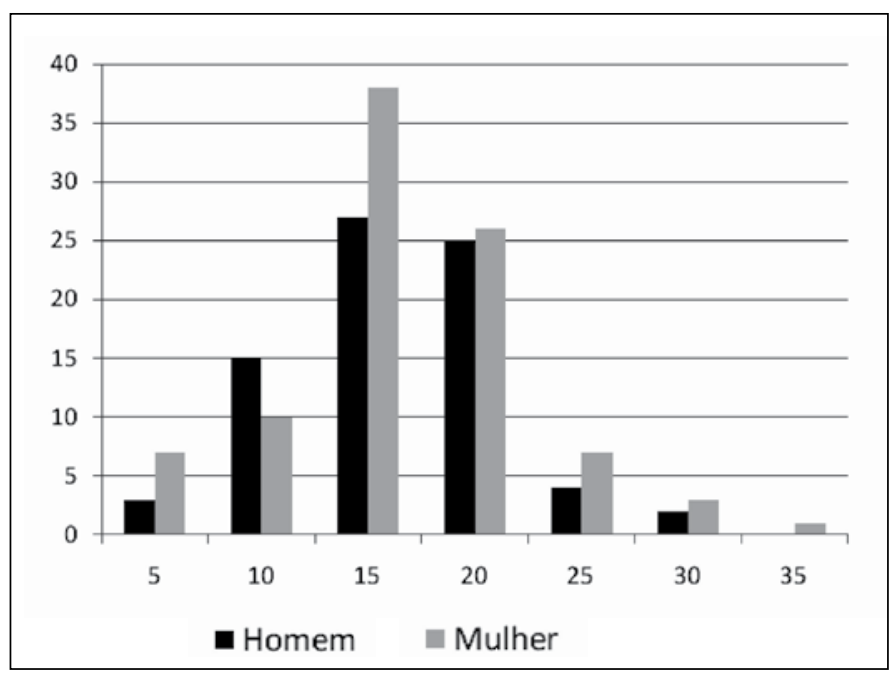

Figura 2. Distribuição dos pacientes por gênero e faixas de idade.

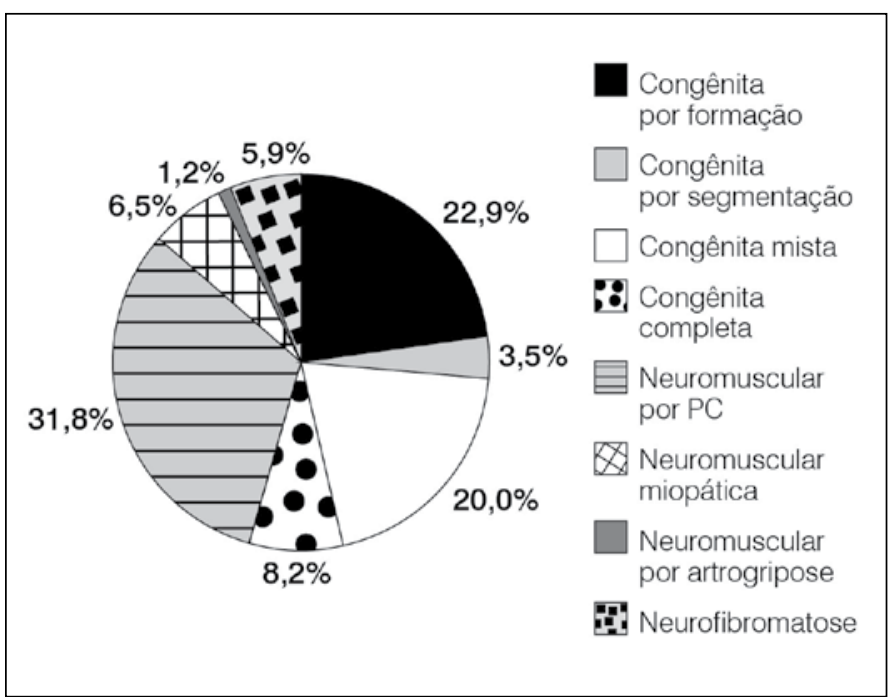

Figura 3. Distribuição das deformidades conforme a etiologia.

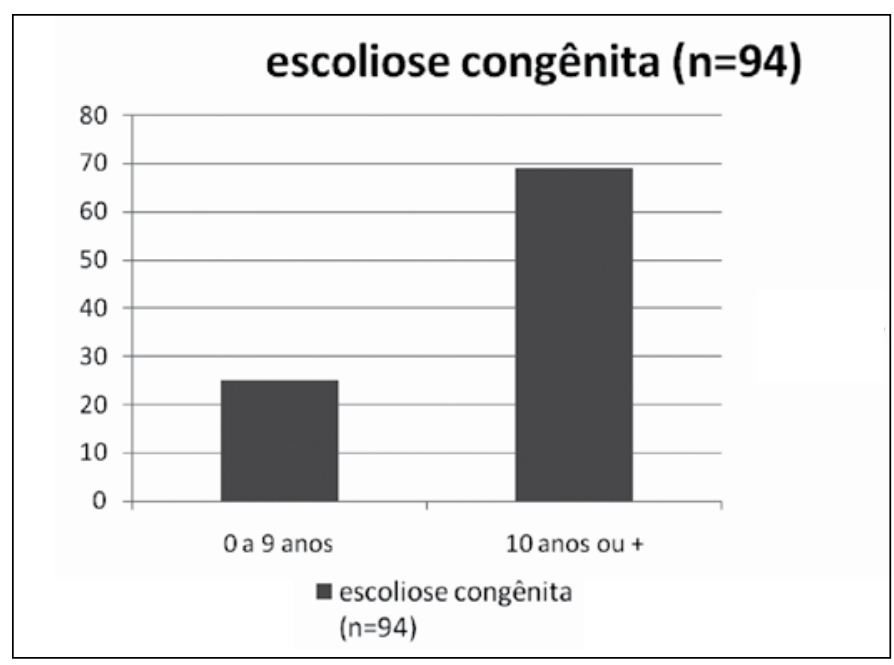

Figura 4. Distribuição das deformidades congênitas por idade.

\section{DISCUSSÃO}

Historicamente, as deformidades causadas por PC apresentam resultados insatisfatórios no tratamento conservador utilizando-se de coletes pelo fato das curvas serem, na maioria das vezes, rígidas e pela baixa adesão dos pacientes ${ }^{11}$. Por este motivo, a fusão cirúrgica, especialmente em pacientes com esqueleto imaturo, tem sido utilizada para prevenir deformidades progressivas e dolorosas que podem interferir na deambulação, balanço coronal-sagital ou levar a outras alterações como úlcera de decúbito ou comprometimento cardiopulmonar $^{12,13}$. Nos últimos 10 anos, estudos demonstraram que, diferentemente da escoliose idiopática, adultos com PC evoluíram com progressão da deformidade, mesmo que inicialmente apresentassem curvas relativamente pequenas ${ }^{14}$. Atualmente, assume-se que exista uma correlação entre a progressão da curva e o déficit funcional ${ }^{15}$.

Já Winter et al. ${ }^{10}$ concluiram que a evolução das curvas congênitas variavam de acordo com o tipo de malformação, contrariando resultados obtidos anteriormente por outros autores que acreditavam que a evolução das escolioses congênitas era de uma maneira geral benigna. Já McMaster e Ohtsuka ${ }^{16}$ estudaram a história natural de 251 pacientes portadores destas deformidades e determinaram o risco de progressão de acordo com o tipo de malformação, idade de surgimento (antes ou depois dos 10 anos), local da deformidade e quantidade de vértebras acometidas (simples ou múltipla). Nos indivíduos com idade inferior a 10 anos, notou-se uma progressão média de 5,0 graus nas curvas torácicas e 6,0 graus nas toracolombares, respectivamente. Já nos tipos mais agressivos como é o caso da hemivertebra com barra óssea contralateral, verificou-se progressão de 10,0 graus por ano nesta mesma faixa etária. Atualmente, sabe-se mais sobre anomalias que acarretam em escolioses congênitas do que quando comparado a qualquer outra etiologia de deformidades na coluna ${ }^{17}$

Quanto às escoliose congênitas, nos defeitos de formação, a progressão varia de acordo com o tipo de anomalia (hemivertebra ou vértebra em cunha), região da coluna acometida, idade do paciente e número de curvas. Escolioses congênitas torácicas altas com uma hemivertebra progridem de 1,0 a 2,5 graus por ano e causam defeito cosmético importante e desnivelamento de ombros, enquanto que esse mesmo tipo de malformação quando acomete a coluna torácica baixa progride entre 2 e 3 graus por ano. O local de maior progressão é na transição toracolombar. Os riscos de progressão nos defeitos de segmentação dependem da extensão da barra óssea e sua localização na coluna. 
Na coluna torácica variam de 2,0 a 6,0 graus por ano, na transição toracolombar entre 6,0 e 9,0 graus e na região lombar em média 5,0 graus por ano. Os defeitos mistos geralmente são aqueles mais rapidamente progressivos, principalmente quando existe um defeito de formação associado a um de segmentação contralateral. Geralmente as curvas congênitas são únicas mas, como nos outros tipos de escolioses, apresentam curvas secundárias e até terciárias que posteriormente se estruturam de acordo com a maturidade esquelética ${ }^{15}$.

\section{CONCLUSÃO}

No hospital terciário de alta complexidade analisado neste estudo existe uma considerável lista de pacientes portadores de deformidades na coluna vertebral que aguardam em fila de espera para tratamento cirúrgico por um período de tempo muitas vezes bastante prolongado. Este fato acarreta em um problema social de difícil manuseio e quando somadas, escoliose neuromuscular e escoliose congênita são responsáveis pela grande maioria dos casos que aguardam cirurgia.

\section{REFERÊNCIAS}

1. Parke WW, Bono CM, Garfin SR. Applied anatomy of the spine. In: Herkowitz HN, Garfin SR, Eismont FJ, Bell GR, Balderston RA Herkowitz HN. The spine. 5th ed. Philadelphia: Sauders/Elsevier; 2006. p.16-XX.

2. Stokes IA, Bigalow LC, Moreland MS. Measurement of axial rotation of vertebrae in scoliosis. Spine (Phila Pa 1976). 1986;11(3):213-8.

3. Newton PO, Wenger DR. Escolioses idiopática e congenital. In: Morrissy RT, Weinstein SL, editoris. Ortopedia pediátrica de Lovell e Winter. 5a. ed. Barueri, SP: Manole; 2005. p. 738-XX.

4. Inoue S, Shinoto A, Ohki I. The moire topography for early detection of scoliosis and evaluation after surgery. In: Combined meeting of SRS and Japanese SRS. Kyoto, Japan; 1977.

5. Lonstein JE, Bjorklund S, Wanninger MH, Nelson RP. Voluntary school screening for scoliosis in Minnesota. J Bone Joint Surg Am. 1982;64(4):481-8.

6. Willner $S$, Udén A. A prospective prevalence study of scoliosis in Southern Sweden. Acta Orthop Scand. 1982:53(2):233-7.

7. King HA. Idiopathic scoliosis. In: Herkowitz HN, Garfin SR, Eismont FJ, Bell GR, Balderston RA Herkowitz HN, editors. The spine. 5th ed. Philadelphia: Sauders/Elsevier; 2006; p. 520-XX

8. Edgar MA. The natural history of unfused scoliosis. Orthopedics. 1987;10(6):931-9.

9. Newton PO, Wenger DR. Escolioses idiopática e congenital. In: Morrissy RT, Weinstein SL. Ortopedia pediátrica de Lovell e Winter. 5a. ed. Barueri, SP: Manole; 2005. p. 743-XX.
10. Winter, RB, Moe JH, Eilers VE. Congenital scoliosis. A study of 234 patients treated and untreated. Part II: Treatment J Bone Joint Surg Am. 1968;50(1):15-47.

11. Brown JC, Swank S, Specht L. Combined anterior and posterior spine fusion in cerebral palsy. Spine (Phila Pa 1976). 1982;7(6):570-3.

12. Broom MJ, Banta JV, Renshaw TS. Spinal fusion augmented by luque-rod segmental instrumentation for neuromuscular scoliosis. J Bone Joint Surg Am. 1989;71(1):32-44.

13. Lonstein JE, Akbarnia A. Operative treatment of spinal deformities in patients with cerebral palsy or mental retardation. An analysis of one hundred and seven cases. J Bone Joint Surg Am. 1983;65(1):43-55.

14. Thometz JG, Simon SR. Progression of scoliosis after skeletal maturity in institutionalized adults who have cerebral palsy. J Bone Joint Surg Am. 1988;70(9):1290-6.

15. Majd ME, Muldowny DS, Holt RT. Natural history of scoliosis in the institutionalized adult cerebral palsy population. Spine (Phila Pa 1976). 1997;22(13):1461-6.

16. McMaster MJ, Ohtsuka K. The natural history of congenital scoliosis. A study of two hundred and fifty-one patients. J Bone Joint Surg Am. 1982;64(8):1128-47.

17. Erol B, Kusumi K, Lou J, Dormans JP. Etiology of congenital scoliosis. UPOJ. 2002:15:37-42.

18. Marks DS, Qaimkhani SA. The natural history of congenital scoliosis and kyphosis. Spine (Phila Pa 1976). 2009;34(17):1751-5. 